\title{
Surgical Resection with Boyd Technique for Metastatic Ewing Sarcoma of the Bone Plus Docetaxel/Gemcitabine, Associated with Improved Outcomes in Tumor Activity.
}

\author{
Maria L Cortes ${ }^{1}$, Veronica Salais Michaus ${ }^{1^{*}}$, Maria F Loya ${ }^{2}$, Alexandro Franco ${ }^{3}$, Jesus J Suarez ${ }^{4}$ \\ 1(Principal Author) Hospital General de Ciudad Juarez, \\ Division of Traumatology and Orthopedics
}

${ }^{1 *}$ (Principal Author and correspondence), Hospital General de Ciudad Juarez, Division of Internal Medicine/Oncology

${ }^{2}$ (Co-Author) Hospital General de Chihuahua "Dr. Salvador Zubiran Anchondo" Division of Internal Medicine

\author{
${ }^{*}$ Corresponding author \\ Veronica Salais Michaus, Internal Medicine Resident, Division of Internal \\ Medicine/Oncology, Hospital General de Ciudad Juarez, Avenida Paseo Triunfo \\ de la Republica, 32340 Ciudad Juarez, Chihuahua
}

Submitted: 04 July 2020; Accepted: 11 July 2020; Published: 22 July 2020
${ }^{3}$ (Co-Author) Hospital General de Chihuahua "Dr. Salvador Zubiran Anchondo” Division of Internal Medicine

${ }^{4}$ (Co-Author) Hospital General de Ciudad Juarez, Division of Traumatology and Orthopedics

\begin{abstract}
Ewing sarcoma is a malignant bone tumor that mainly affects children, adolescents and young adults with more than 1.5 cases per million worldwide. Approximately 20-25\% of patients present metastatic disease at the diagnosis, that is often resistant to intensive therapy. We present the case of a 19-year-old male with history of epilepsy who started his condition with weight loss, increased volume, pain, swelling after receiving surgical treatment due to a left distal femur fracture, showing tomographic evidence of a 50-cm tumor with multiple lung lesions, so disarticulation was performed with the Boyd technique, obtaining histopathological result of Ewing's Sarcoma, and was subsequently sent to the medical oncology service for follow-up and adjuvant treatment with significant clinical and radiological improvement in pulmonary metastatic activity.
\end{abstract}

Key Words: Ewing Sarcoma, Pelvic Limb Pain, Pathological Fracture, Metastasis, Multidisciplinary Treatment.

Abbreviations

ES: Ewing's sarcoma

DOX: Doxorubicin

CPM: Cyclophosphamide

VCR: Vincristine

ACT-D: Actinomycin-D

IFO: Ifosfamide

ETO: Etoposide

\section{Introduction}

Ewing's sarcoma is a malignant bone tumor that occurs predominantly in the pelvis, femur, tibia and ribs or as a soft tissue tumor located in the chest wall, gluteal muscle, pleural cavities, and cervical muscles, with more than 1.5 cases per million worldwide. Approximately $20-25 \%$ of patients have metastases at diagnosis that are often resistant to intensive therapy [1]. Ewing's sarcoma (ES) is the second most common malignant primary bone tumor in children and adolescents, with a peak incidence at the age of 15 years; boys and men are slightly more affected than girls and women (sex ratio of 3:2) The origin of this tumor was unclear until recently, when electron microscopic and immunohistochemical analyzes suggested that it is of neurogenic origin, ES tumors often express a balanced translocation involving the EWS gene on chromosome 22 and a member of the ETS family of transcription factors [2-6]. As with other primary bone sarcomas, pain is the most common initial symptom of patients with Ewing's sarcoma of bone. As the tumor destroys bone, patients may notice a deep, dull, aching pain in the involved region or extremity, it is not uncommon for patients to present with fever or weight loss, which in the presence of bone pain may lead the physician into misdiagnosing the cause as osteomyelitis [7,8]. Histologically, Ewing's sarcoma appears as sheets of homogenous densely packed small round blue cells. They have a high nuclear to cytoplasm ratio and the nucleus is associated with fine granular chromatin and pinpoint nucleoli. The cytoplasm typically has few or small organelles and abundant glycogen [9]. In ES of the bone, plain radiographs exhibit permeative and infiltrative destruction of the affected bone. In addition, an onion skin-like appearance and spiculae are indicative of periosteal reactions [10].

Current standard of localized or regional treatment for patients with ES includes systemic chemotherapy and surgery and/or radiation therapy for local control [11]. Due to improvements in intensive chemotherapy, the prognosis of ES patients has improved markedly. The current chemotherapy protocols used to treat ES include various combinations of the following six drugs: doxorubicin (DOX), cyclophosphamide (CPM), vincristine (VCR), actinomycin-D (ACT-D), ifosfamide (IFO), and etoposide (ETO) [2]. Gemcitabine in combination with docetaxel was found to be well tolerated and demonstrated antitumor activity in children and ado- 
lescents with recurrent or refractory disease [12].

Whether surgical excision of the primary bone tumor improves the survival of patients who present with metastatic ES is unknown, there are no large series covering this issue. Some authors advise amputation as the "gold standard" treatment $[13,14]$. Therefore, local surgery is an appropriate treatment for patients with metastatic ES. Raciborska et al. reported that treatment of isolated lung metastases may have a role in improving prognosis in patients with ES. Letourneau et al $[15,16]$.

\section{Materials and Methods}

We present the case of a 19-year-old male patient, with a history of epilepsy, who suffers a diaphyseal fracture of his left distal femur, an open reduction and internal fixation were performed using an anatomical plate for the distal femur. The patient comes to the consultation because after the surgical intervention, the pain becomes incapacitating and presents a progressive increase in volume. Upon physical examination (Figure 1), a collateral venous network is observed, he presents hyperthermia in the thigh and a palpable mass of approximately $50 \mathrm{~cm}$, which is painful, of rigid consistency and is fixed to deep planes, in addition to this, the patient reports loss of $10 \mathrm{~kg}$ weight in a month, therefore, we requested an X-ray (Figure 2) and pelvic limb tomography (Figure 3 ) in the face of findings compatible with a tumor lesion, a chest tomography was also requested to perform a scan, (Figure 4) finding lesions compatible with metastatic pulmonary activity. When faced with advanced disease, the left pelvic limb was disarticulated using the Boyd technique, obtaining a histopathological diagnosis of Ewing's Sarcoma and infiltration of muscle fibers and atrophy (Figure 5). With adequate post-operative evolution and no data of infection in the surgical wound, he was referred to the medical oncology service for treatment with adjuvant chemotherapy based on Docetaxel and Gemcitabine; currently in the fourth cycle, clinically and radiologically corroborating significant improvement in dyspnea and lung lesions as well as improvement in respiratory symptoms and quality of life.

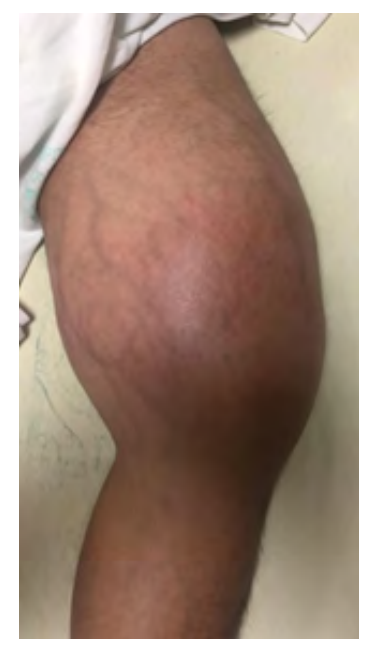

Figure 1: Tumor in the left pelvic limb on physical examination.
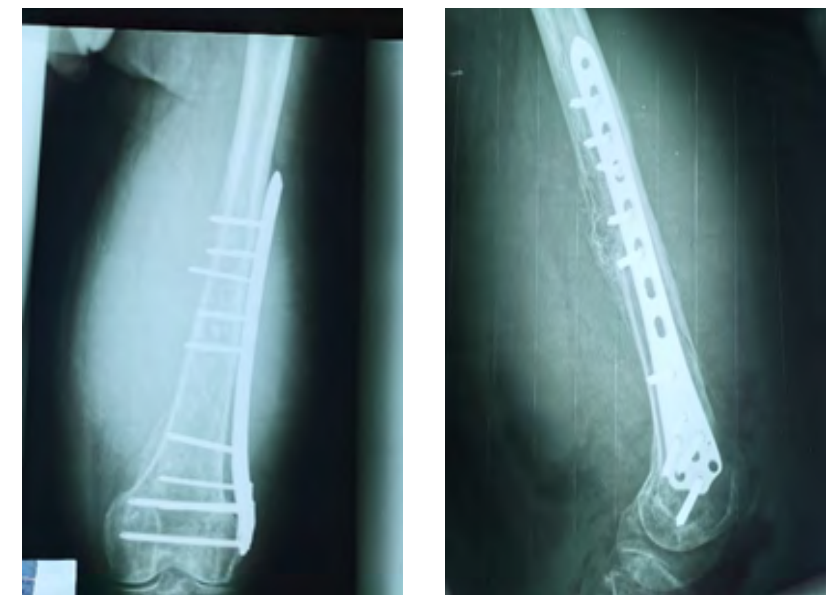

Figure 2: A: Soft tissue tumor B: Lytic image with poorly defined edges, with permeative and moth-eaten pattern, spiculated and multilaminar discontinuous periosteal reaction.

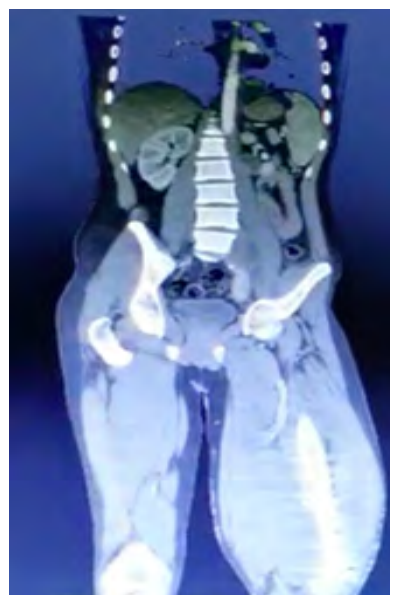

Figure 3: Heterogeneous tumor with irregular, poorly defined edges, 198X240 mm at the level of muscle structures with infiltration and bone remodeling that breaks the cortex, predominantly in the middle and lower third of the femur

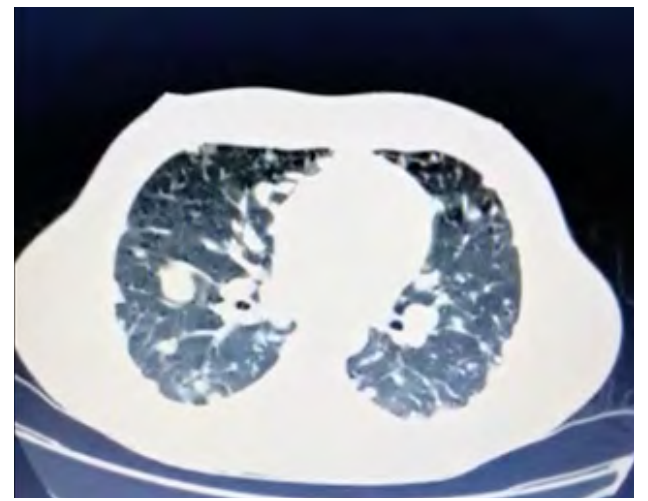

Figure 4: Lung parenchyma with multiple diffuse nodular lesions in both hemithorax and infiltrating mediastinal and parahilar adenomegalies that form $32 \mathrm{~mm}$ conglomerates with hypodensity suggestive of necrosis. 

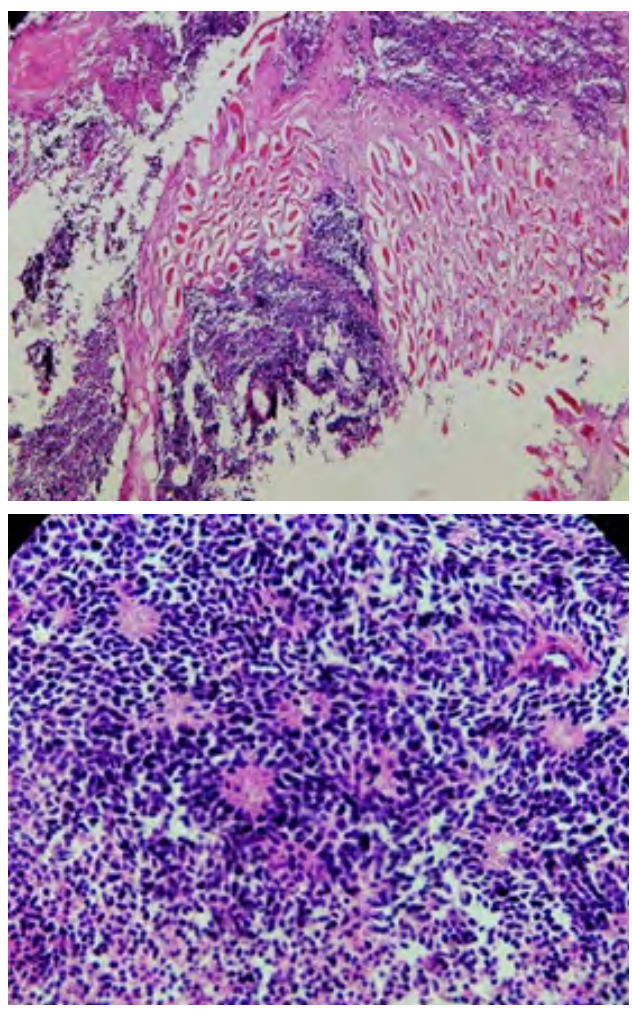

Figure 5: A: Histological section with hematoxylin-eosin stain, in which tumor infiltration of atrophic muscle fibers is observed. B: Homer-Wright type rosette formation in Ewing's sarcoma.

Results

The surgical technique is described below. The subcutaneous cellular tissue is dissected until the femoral neurovascular bundle is located, which is ligated and cut, the sartorius and rectus femoris muscles are disengaged from the anterosuperior and anteroinferior iliac spine respectively and are retracted distally, the pectineus muscle is dissected distal to the pubis, external rotation of the limb is performed and the psoas muscle is removed from the lesser trochanter. The adductor muscles of the hip and the gracilis muscle in their proximal insertions are dissected, the obturator artery is located, ligated and cut. Subsequently, the limb is rotated internally, the gluteus minimus, medius, and the most distal fibers of the gluteus maximus are disengaged from the rough line and the sciatic nerve is then located to perform ligation and cutting. The external rotators of the hip are detached from their origin. The section of the articular capsule and the round ligament are carried out, the gluteal flap is carried towards the anterior region, the distal part of these muscles is sutured at the insertion site of the pectineus and the obturator (Figure 6).

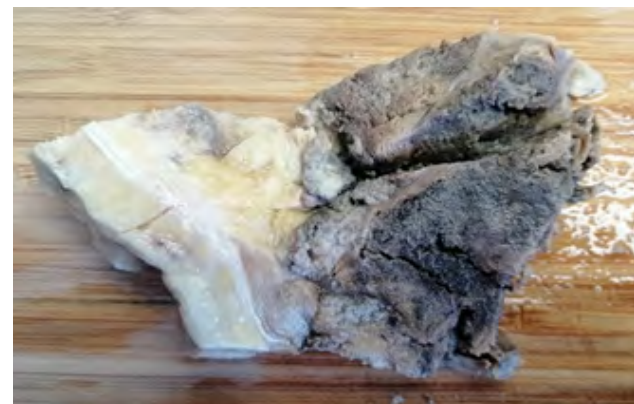

Figure 6.- Macroscopic piece in cross section where infiltration to muscle fibers is observed.
Discussion \& Conclusion

Ewing's sarcoma is an entity that occurs especially in a young population, so it is of most importance to make the correct diagnostic approach in the presence of pathological or suggestive fractures in this type of patient due to the high index of presence of metastases at the time of diagnosis, likewise, perform a multidisciplinary treatment and a correct individualized surgical approach to each patient, in which the objective is the total resection of the tumor, as well as a higher quality of life. In the case presented, it was decided to perform a disarticulation with the Boyd technique

Acknowledgements: We especially want to thank the pathology service of the Hospital General de Ciudad Juarez for their valuable support in the work and processing of the sample as well as for the collaboration of all the staff in this case report and review.

\section{References}

1. Nathalie Gaspar, Douglas S Hawkins, Uta Dirksen, Ian J Lewis, Stefano Ferrari, et al. (2015) Ewing Sarcoma: Current Management and Future Approaches through Collaboration. J Clin Oncol 33: 3036-3046.

2. Grier HE, Krailo MD, Tarbell NJ, Link MP, Fryer CJH, et al. (2003) Addition of Ifosfamide and Etoposide to Standard Chemotherapy for Ewing's Sarcoma and Primitive Neuroectodermal Tumor of Bone. N Engl J Med 348: 694-701.

3. Ginsberg JP, Goodman P, Leisenring W, Ness KK, Meyers PA, et al. (2010) Long-term survivors of childhood Ewing sarcoma: Report from the childhood cancer survivor study. J Natl Cancer Inst 102: 1272-1283.

4. Jawad MU, Cheung MC, Min ES, Schneiderbauer MM, Koniaris LG, et al. (2009) Ewing sarcoma demonstrates racial disparities in incidence-related and sex-related differences in outcome: an analysis of 1631 cases from the SEER database, 1973-2005. Cancer 115: 3526-3536.

5. Jaffe R, Santamaria M, Yunis EJ, Tannery NH, Agostini RM, et al. (1984) The Neuroectodermal Tumor of Bone. Am J Surg Pathol 8: 885-898.

6. De Alava E, Lessnick SL, Sorensen PH (2013) Ewing sarcoma. In: Fletcher CDM, Bridge JA, Hogendoorn PCW, Mertens F, editors. WHO classification of tumours of soft tissue and bone. Lyon: WHO Press 305-309.

7. Patricio MB, Vilhena M, Neves M, Raposo S, Catita J, et al. (1991) Ewing's sarcoma in children: twenty-five years of experience at the Instituto Portugês de Oncologia de Francisco Gentil (I.P.O.F.G.). J Surg Oncol 47: 37-40.

8. Widhe B, Widhe T (2000) Initial symptoms and clinical features in osteosarcoma and Ewing sarcoma. J Bone Joint Surg Am 82: 667-674.

9. Suh CH, Ordóñez NG, Hicks J, Mackay B (2002) Ultrastructure of the Ewing's sarcoma family of tumors. Ultrastruct Pathol 26: 67-76.

10. Esiashvili N, Goodman M, Marcus RB (2008) Changes in incidence and survival of Ewing sarcoma patients over the past 3 decades: Surveillance Epidemiology and End Results data. J Pediatr Hematol Oncol 30: 425-430.

11. Marco RAW, Gentry JB, Rhines LD, Lewis VO, Wolinski JP, et al. (2005) Ewing's sarcoma of the mobile spine. Spine 30: 769-773.

12. Leu KM, Ostruszka LJ, Shewach D, Mark Zalupski, Vernon Sondak, et al. (2004) Laboratory and clinical evidence of synergistic cytotoxicity of sequential treatment with gemcitabine followed by docetaxel in the treatment of sarcoma. J Clin On- 
col 22: 1706-1712.

13. Lee CY, Yen CC, Yen HJ, Shiau CY, Chao TC, et al. (2016) Outcomes of 50 patients with Ewing sarcoma family of tumors treated at a single institution in Taiwan. Medicine 95: e3830.

14. Mikel SJ, Julio D, Pablo Diaz de R, Luis S (2008) Limb Salvage in Ewing's Sarcoma of the Distal Lower Extremity. Foot \& Ankle International 29: 22-28.
15. Raciborska A, Bilska K, Rychlowska-Pruszynska M, Marek Duczkowski, Agnieszka Duczkowska, et al. (2016) Management and follow-up of Ewing sarcoma patients with isolated lung metastases. J Pediatr Surg 51: 1067-1071.

16. Letourneau PA, Shackett B, Xiao L, Jonathan Trent, Kuo Jen Tsao, et al. (2011) Resection of pulmonary metastases in pediatric patients with Ewing sarcoma improves survival. J Pediatr Surg 46: 332-335.
Copyright: (2020 Veronica Salais Michaus., This is an open-access article distributed under the terms of the Creative Commons Attribution License, which permits unrestricted use, distribution, and reproduction in any medium, provided the original author and source are credited. 